\title{
Evaluación de clínica y calidad de vida con catéteres ureterales de tipo doble pig-tail
}

\author{
Vega Vega A, García Alonso D, García Alonso CJ.
}

Hospital de León. León.

Actas Urol Esp. 2007;31(7):738-742

\section{RESUMEN}

EVALUACIÓN DE CLÍNICA Y CALIDAD DE VIDA EN PACIENTES CON CATÉTERES URETERALES TIPO DOBLE PIG-TAIL.

Objetivos: Valoración de la prevalencia de la clínica asociada a los catéteres ureterales tipo doble pig-tail y de su impacto en la calidad de vida a través de un cuestionario.

Material y métodos: 100 pacientes portadores de doble pig-tail cumplimentaron el cuestionario. Este incluye preguntas sobre clínica urinaria: disuria, polaquiuria, tenesmo, urgencia, incontinencia, hematuria y dolor o malestar provocado por el catéter durante las actividades normales del paciente. También contestaron el tes IPSS y una cuestión sobre la calidad de vida. El dolor se valoró de 1 a 10 mediante una escala visual analógica.

Resultados: Se valoró 46 varones y 54 mujeres, con una edad media de 54 años. El tiempo medio de colocación del catéter fue de 6,5 semanas. La colocación se debió en un 40\% al tamaño de la litiasis, previo a la litotricia, en un $33 \%$ por obstrucción, en un $25 \%$ por infección y en el resto fue postquirúrgico. El dolor de la colocación sin anestesia general se produjo en el 95\% de los pacientes. En un 89\% de los pacientes la presencia del catéter produjo clínica, el orden de frecuencia fue: nicturia, polaquiuria, tenesmo, disuria, urgencia, hematuria e incontinencia. En un 73 \% les produjo dolor, en un 52\% éste se localizó en el flanco y en el $47 \%$ en la vejiga. Un $60 \%$ de los pacientes se consideraron insatisfechos con su calidad de vida con el catéter.

Conclusiones: La clínica urinaria y el dolor asociados a los catéteres ureterales tipo doble pig-tail interfiere con las actividades de la vida diaria y reduce la calidad de vida en un $60 \%$ de los pacientes.

Palabras clave: Catéter ureteral. Calidad de vida. Uréter.

\section{ABSTRACT \\ CHARACTERIZATION OF URINARY TRACT SYMPTOMS AND QUALITY OF LIFE IN PATIENTS WITH DOUBLE-PIG-TAILED URETERAL STENTS}

Objectives: To prospectively assess the prevalence of urinary tract symptoms caused by indwelling ureteral stents and how they affect their quality of life using an specific questionnaire.

Material and methods: A total of 100 patients with unilateral indwelling ureteral stents were asked to complete an specific questionnaire that include questions on urinary symptoms: dysuria , frequency of urination, incomplete empting, urgency, incontinence, haematuria and bother or pain associated with urinary stents affecting the daily activities of the patients. They were also asked to compete International Prostate Symptoms Score questionnaire and a question on quality of life. Pain was assessed from 0 to 10 using a VAS (visual analogic scale).

Results: 46 men and 54 women, mean age 54 years, completed the study. Mean time after stent insertion was 6.5 weeks. The cause of the insertion was in $40 \%$ due to the size of the stone, previous to ESWL, 33\% due to urinary tract obstruction, 25\% due to infection, and the others after a surgical procedure. 95\% of patients with stent positioning without general anesthesia complaint of pain during the procedure. 89\% of patients reported bothersome urinary symptoms, named in order of frequence: nycturia, frequence voiding, incomplete empting, dysuria, urgence, haematuria and incontinence. More than $70 \%$ of patients experienced pain that impaired daily activities, in 52\% pain was located at the flank and in $47 \%$ at the bladder. $60 \%$ of patients signalled that they were not satisfied with their quality of life with the indwelling catheter.

Conclusions: Urinary symptoms and pain related to indwelling double pig tailed ureteral stens affect the daily activities and reduces the quality of life of $60 \%$ of patients.

Keywords: Ureteral stents. Ureter. Quality of life. 
$\mathrm{E}^{\prime}$ 1 uso de los catéteres ureterales de tipo doble pig-tail es una práctica habitual en la urología como prevención de complicaciones, estando asociado en la mayoría de los casos con la presencia de litiasis y de dificultades en el drenaje en el aparato urinario. Las indicaciones para la colocación del stent son ${ }^{1,2}$ :

- disminución de incidencia de complicaciones en receptores de trasplantes renales.

- presencia de litiasis mayor de $2 \mathrm{~cm}$ previo a la litotricia extracorpórea para favorecer la eliminación de litiasis y evitar la formación de calles litiásicas.

- manejo de obstrucción ureteral extrínseca o maligna.

- infección con dificultad de drenaje.

- cólicos incoercibles que no ceden con tratamiento médico.

- tras intervenciones quirúrgicas como: endopielotomía, pieloplastia, incisión de estenosis ureteral, ureteroscopia complicada, tratamiento incompleto de litiasis, uso prolongado de ureteroscopia flexible intrarenal, uso de vaina de acceso ureteral.

Cuando existen litiasis, el mayor beneficio del uso del stent es la prevención de complicaciones por una posible obstrucción ureteral al paso de los fragmentos de litiasis desde el riñón a la vejiga a través del uréter. El material empleado es un material flexible para evitar el disconfort e incrustaciones (poliuretano, silicona y polímeros) ${ }^{3}$. La presencia del stent se asocia con una gran variedad de síntomas, siendo principalmente a nivel del tracto urinario inferior ${ }^{4,5}$. En la vejiga produce clínica irritativa con sensación de disconfort, además del riesgo de migración, reflujo vesico-ureteral e incrustación en la superficie del stent ${ }^{2,5}$.

Existen pocos estudios de la valoración de la clínica y de cómo afecta a la calidad de vida del paciente $^{6}$. En nuestro estudio pretendemos la valoración de la clínica urinaria mediante un cuestionario específico, en el que también se incluyen preguntas sobre calidad de vida y dolor, con su baremación mediante el IPSS (Internacional Prostate Symptom Store) y la VAS (Escala Visual Analógica). Analizamos la prevalencia de la clínica urinaria, con su impacto en la calidad de vida en los pacientes portadores de catéter doble $\mathrm{J}$, los cuales en ocasiones ponemos tan ale- gremente y no somos conscientes de los efectos que tienen en los pacientes, tanto su colocación como su presencia.

\section{MATERIAL Y MÉTODOS}

Se pidió el consentimiento para el estudio a 100 pacientes consecutivos portadores de catéter tipo doble pig-tail unilateral. Los pacientes cumplimentaron un cuestionario que incluye preguntas sobre clinica urinaria: polaquiuria, disuria, urgencia, tenesmo, incontinencia, hematuria, nicturia, necesidad de toma de analgésicos, empeoramiento con ejercicio; preguntas sobre dolor provocado por el catéter tanto a nivel del flanco como a nivel vesical, en descanso, al andar $\mathrm{y}$ al orinar; completaron el estudio IPSS y una cuestión sobre calidad de vida. Además se preguntó por el dolor en la colocación y si esta fue con anestesia local, sedación o anestesia general. También se recogió la edad, sexo y tiempo de colocación del catéter. El dolor se valoró mediante escala visual analógica (VAS) de 0 a 10.

El catéter ureteral de tipo doble pig-tail que portaban los pacientes en todos ellos tenía un tamaño de 24 a $28 \mathrm{~cm}$ dependiendo de la altura del paciente, de $6 \mathrm{~F}$ de calibre y de composición de poliuretano, todos ellos de la casa Cook.

Los 100 pacientes portadores de catéter ureteral de tipo doble pig-tail tenían litiasis, 46 varones y 54 mujeres, con una edad media de 54 años. El tiempo medio desde la colocación del catéter fue de 6,5 semanas. En 42 pacientes la colocación fue en el lado derecho y en 58 en el lado izquierdo. En un 35\% la colocación se debió al tamaño de la litiasis a tratar mediante litotricia extracorpórea, en un 35\% por obstrucción por la litiasis, en un $25 \%$ por presencia de infección, y en el $5 \%$ restante fue postquirúrgico y por presencia de estenosis.

\section{RESULTADOS}

Dolor a la colocación del catéter: Tabla 1: se produjo dolor en casi todos los pacientes en los que la colocación del catéter se hizo con anestesia local, con lubricante con lidocaína, siendo esta colocación muy dolorosa, con una media de dolor de 6,8 según escala VAS de dolor, en los pacientes en los que se hizo sedación, aunque la mayoría de ellos tuvo dolor este fue mucho más 
Tabla 1

Valoración de dolor mediante VAS (Escala Visual Analógica) dependiendo del tipo de anestesia empleado.

\begin{tabular}{lccc}
\hline Colocación catéter & Anestesia local & Sedación & Anestesia general \\
\hline \% pacientes & 56 & 8 & 36 \\
Rango dolor VAS & $0-10$ & $0-7$ & 0 \\
Media dolor VAS & $6,8 \pm 3,07$ & $3,6 \pm 2,71$ & 0 \\
\% pac. con dolor & $95 \%$ & $87 \%$ & 0 \\
\hline
\end{tabular}

\section{DISCUSIÓN}

Los resultados de nuestro estudio indican que la presencia de catéteres ureterales doble pig tail en los pacientes se asocia en un alto porcentaje, en el 90\% de los casos, con una gran variedad de clínica urinaria, así como con la pre-

moderado, con una media de 3,6 sobre 10 en la escala VAS.

Clínica Urinaria: Tabla 2: no se produjo clínica urinaria en un $10 \%$ de los pacientes portadores de stent. La clínica predominante fue la nicturia y la polaquiuria. En más de la mitad de los casos se produjo tenesmo y urgencia; disuria en uno de cada 2 , y por orden de frecuencia la clínica fue de hematuria, incontinencia y sólo en un $5 \%$ hubo como complicación infección de orina. En un $20 \%$ de los pacientes se precisó analgesia y la clínica aumentó durante el ejercicio.

Dolor: Tabla 3. En 3 de cada 4 pacientes la presencia del pig-tail produjo algún grado de dolor, en unos casos en el flanco y en otros en la vejiga. En las 2 localizaciones, el dolor fue más frecuente al andar, y su grado muy leve.

IPSS: Tabla 4 la mayoría de los pacientes presentaron nicturia y polaquiuria, quejándose de sensación de no vaciar por completo la vejiga y de urgencia miccional, la imposibilidad de aguantarse las ganas de orinar.

Calidad de vida: Tabla 5. La mayoría de los pacientes se encuentran insatisfechos-mal por la presencia del catéter.

Tabla 2. Presencia o ausencia de clínica urinaria en pacientes portadores de catéter tipo doble pig-tail

\begin{tabular}{lcc}
\hline Clinica urinaria & No (\%) & Si (\%) \\
\hline Presencia de clinica & 10 & 90 \\
Polaquiuria & 39 & 61 \\
Disuria & 53 & 47 \\
Urgencia & 46 & 54 \\
Incontinencia & 75 & 25 \\
Tenesmo & 45 & 55 \\
Sangre macroscópica & 64 & 36 \\
Nicturia & 36 & 64 \\
Infección & 95 & 5 \\
Precisa analgesia & 82 & 18 \\
Aumenta con ejercicio & 82 & 18 \\
\hline
\end{tabular}

sencia de dolor. Estos efectos secundarios tienen un gran impacto negativo en la calidad de vida de los pacientes ${ }^{6}$. Para la valoración de la clínica y efectos secundarios producidos por los catéteres, al no existir un cuestionario estandarizado, en nuestro estudio hemos valorado en primer lugar la presencia de dolor en la colocación de los catéteres, posteriormente se ha valorado la presencia o ausencia de clínica urinaria, basado en la descripción de la clínica presente en los pacientes con catéter de tipo doble pig-tail ${ }^{4,5}$. La presencia de dolor se midió por escala visual analógica (VAS) de uno a 10, y su localización en flanco o a nivel vesical. Posteriormente hemos usado el IPSS para valoración de la clínica a nivel de tracto urinario inferior. Además hemos añadido una pregunta sobre la calidad de vida de los pacientes.

La colocación del catéter presenta grandes diferencias en el dolor que refieren los pacientes según sea su colocación con anestesia general, el $100 \%$ de los pacientes no presenta dolor, con la anestesia local o sedación, aunque el porcentaje de pacientes con dolor es similar, 95 y $87 \%$ respectivamente, la cualidad del dolor es completamente diferente, mientras que la media de dolor

Tabla 3. Presencia o ausencia de dolor, localización y valoración en las distintas localizaciones.

\begin{tabular}{lcccc}
\hline Dolor & No (\%) & Si (\%) & & \\
Presencia de dolor & 28 & 72 & & \\
Dolor en flanco & 49 & 52 & & \\
Dolor vesical & 56 & 44 & & \\
\hline Dolor en flanco & No (\%) & Si (\%) & Rango & Media \\
En descanso & 80 & 20 & $0-6$ & $2,66 \pm 1,89$ \\
Al andar & 64 & 36 & $0-8$ & $3,32 \pm 2,11$ \\
Al orinar & 76 & 24 & $0-9$ & $3,61 \pm 1,51$ \\
\hline Dolor en vejiga & No (\%) & Si (\%) & Rango & Media \\
En descanso & 86 & 14 & $0-7,2$ & $3,40 \pm 2,19$ \\
Al andar & 67 & 33 & $0-8$ & $3,17 \pm 1,82$ \\
Al orinar & 71 & 29 & $0-7$ & $3,70 \pm 1,2$ \\
\hline
\end{tabular}


Tabla 4. Valoración IPSS en pacientes portadores de pig-tail.

\begin{tabular}{|c|c|c|c|c|c|c|}
\hline Baremo IPSS & ninguna & $\begin{array}{l}1 \text { vez de } \\
\text { cada } 5\end{array}$ & $\begin{array}{c}1 \text { vez de } \\
\text { cada } 3\end{array}$ & $\begin{array}{l}1 \text { vez de } \\
\text { cada } 2\end{array}$ & $\begin{array}{c}2 \text { vez de } \\
\text { cada } 3\end{array}$ & $\begin{array}{c}\text { CASI } \\
\text { siempre }\end{array}$ \\
\hline Tenesmo & 41 & 11 & 11 & 2 & 2 & 33 \\
\hline Orinar en menos de $2 \mathrm{~h}$. & 23 & 17 & 19 & 7 & 8 & 26 \\
\hline Chorro intermitente & 84 & 5 & 3 & & & 8 \\
\hline Urgencia & 49 & 10 & 9 & 8 & 2 & 22 \\
\hline Chorro flojo & 79 & 3 & 4 & 1 & & 13 \\
\hline \multirow[t]{2}{*}{ Esforzarse al orinar } & 91 & & 1 & 1 & & 7 \\
\hline & ninguna & 1 vez & 2 veces & 3 veces & 4 veces & 5 veces o más \\
\hline nicturia & 22 & 22 & 26 & 20 & 8 & 2 \\
\hline
\end{tabular}

Tabla 5. Calidad de vida de los síntomas urinarios. ¿cómo se sentiría si tuviera que pasar toda su vida orinando como lo hace ahora (con el catéter)?

\begin{tabular}{cccccccc}
\hline Calidad de vida & Encantado & Contento & Satisfecho & Indiferente & Insatisfecho & Mal & Muy mal \\
\hline 4 & 6 & 10 & 20 & 35 & 22 & 3 \\
\hline
\end{tabular}

es de casi 7 , lo cual es un dolor altísimo y difícil de soportar en los pacientes en los que se le coloca el catéter con anestesia local, en los pacientes en los que la colocación es con sedación el dolor tiene una media de 3,5 un dolor leve, claramente soportable. Esto nos indica que debemos de programar sedación o anestesia general para la colocación de un catéter tipo doble pig-tail si valoramos el bienestar del paciente y no queremos producirle un dolor tan intenso que la mayoría de los pacientes no se volvería a dejar otra vez un catéter sin anestesia general o sedación.

La evaluación de la clínica urinaria nos indica que ésta se presenta en un 90\% de los pacientes, con la presencia de nicturia en un $64 \%$, y de polaquiuria en un 61\% de los pacientes. Esta sintomatología, además de ser un efecto secundario irritativo del catéter, puede deberse a la asociación de un aumento de ingesta de líquidos por parte de los pacientes. La irritabilidad vesical del catéter queda de manifiesto con la presencia de tenesmo, sensación de vaciado incompleto de la vejiga en un 55\% de los pacientes, así como en la urgencia miccional, la dificultad para aguantarse las ganas de orinar, en un 54\%. Esta urgencia se convierte en incontinencia, la pérdida de orina sin control voluntario de la misma, en un $25 \%$ de los pacientes, lo cual puede deberse a exacerbar la contractibilidad del detrusor por el estímulo mecánico del stent ureteral sobre las paredes de la vejiga. Se ha culpado a la porción intervesical de la endopróte- sis de los sintomas irritativos ${ }^{7}$.

La presencia de disuria, micción con molestia, se produce en un $47 \%$ de los pacientes con pigtail y ésta no desaparece hasta que no se retira el catéter, claramente relacionado con la estimulación e irritabilidad que produce su presencia en la vejiga, cuanto más tiempo está el catéter presente, más se acentúa. Todo lo contrario sucede en los pacientes en los que se presenta hematuria, ésta es más frecuente en los primeros días de la colocación del catéter, disminuyendo progresivamente según avanza el tiempo desde que se le ha colocado $^{5}$. Este síntoma se produce en un $36 \%$ de los pacientes, y se asocia con ejercicio, andar, correr, etc.

El ejercicio produce un aumento de la clínica en el 18\% de los pacientes. La clínica urinaria es tan molesta que precisa analgesia en un 18\% de los pacientes, lo cual indica que, aunque afecta a la calidad de vida, las molestias son fácilmente soportables. Sólo se ha complicado con infección en vías urinarias altas en un 5\% de los pacientes, los cuales han precisado tratamiento antibiótico para la misma, algunos incluso con ingreso hospitalario.

El dolor estuvo presente en un $72 \%$ de los pacientes, aunque la mayoría lo describen como una molestia, un 52\% de los pacientes presenta dolor en el flanco y en un 44\% de los pacientes el dolor es a nivel vesical. Entre los que presentan dolor lumbar, 36 pacientes, el 69\% el dolor o las 
molestias se producen al andar, en un $45 \%$ este dolor se produce al orinar, probablemente debido al reflujo miccional hacia el riñón por la presencia de la endoprótesis ${ }^{3,7}$, y en un $38 \%$ la presencia del dolor no se relaciona con la actividad, presentando dolor en reposo. El $66 \%$ de los pacientes con dolor a nivel vesical este se presentaba al orinar, producto de la irritación mecánica del catéter, en un $75 \%$ este dolor se producía al andar, también por la irritación del extremo en la vejiga, y en un $32 \%$ este dolor se produce durante el descanso, con independencia de la actividad.

En la baremación IPSS, la clínica más manifiesta es la nicturia, con más de un $50 \%$ de los pacientes que orina más de 2 veces a la noche y lo que refieren también los pacientes es un aumento importante de la frecuencia para orinar. La clínica más molesta para el paciente es la sensación de no vaciar por completo, que en un 33\% de los pacientes se produce cada vez que van a orinar. Otro síntoma muy molesto para los portadores del pig-tail es la urgencia, la imposibilidad de aguantarse las ganas de orinar todas las veces por la irritabilidad y estimulación del detrusor por la porción de la endoprótesis en la vejiga.

Respecto a la pregunta sobre la calidad de vida durante el tiempo en el que el paciente lleva el catéter ureteral, en un $60 \%$ de los pacientes se produce alteración en su calidad de vida, presentando el $25 \%$ del total de los pacientes una afectación importante con una tolerancia mala o muy mala de la presencia del catéter.

Una proporción importante de los paciente presenta aumento de frecuencia y urgencia, con/sin incontinencia, síntomas descritos en la vejiga hiperactiva, pudiendo exacerbar una inestabilidad del detrusor subclínica. En los pacientes en los que se produce dolor suprapúbico, hematuria y aumento de frecuencia, el tratamiento con antiespasmódicos como oxibutina o tolterodina reducen estos síntomas y lo hacen más tolerable ${ }^{4}$.

\section{CONCLUSIONES}

La disponibilidad de los stent ureterales y sus aplicaciones ofrecen una solución rápida y simple a problemas graves debidos a la obstrucción ureteral. A pesar de ser esencial y de gran utilidad en la urología no está exento de efectos secundarios. La colocación del stent con anestesia local, práctica muy habitual en nuestro medio, ha de evitarse por el gran disconfort y dolor que produce en el paciente, siendo adecuada su colocación con anestesia general o sedación. El 90\% de los pacientes con stent ureteral presenta clínica urinaria, siendo la más frecuente nicturia, aumento de frecuencia, tenesmo, disuria y hematuria. El dolor o algún tipo de molestia se produce en un $72 \%$ de los pacientes, en un $52 \%$ este se localiza a nivel lumbar y en un $44 \%$ a nivel suprapúbico. Los pacientes consideran que la presencia del catéter reduce su calidad de vida en un $60 \%$ de los ellos. Es importante cuando se coloca un catéter al enfermo informarle de los efectos secundarios que produce, hacerle un seguimiento para valorar la clínica y en los casos necesarios tratarle.

\section{REFERENCIAS}

1. Chew BH, Knudsen BE, Denstedt JD. The use of stents in contemporary urology. Curr Opin Urol. 2004;14(2): 111-115.

2. Saltzman B: Ureteral stent indications, variations, and complications. Urol Clin North Am. 1988; 15(3):481-491.

3. Thomas R. Indwelling ureteral stens: impact of material and shape on patient comfort. J Endourol. 1993;7(2): 137-140.

4. Irani J, Siquier J, Pires C, Lefebvre O, Dore B, Auber J. Symptom charactheristics and development of tolerance with time inpatients with indwelling double pigtail ureteric stents. BJU Int. 1999;84(3):276-279.

5. Joshi HB, Okeke A, Newns N, Keeley FX, Timoney AG. Characterization of urinary symptoms in patients with ureteral stents. Urology 2002;59(4):511-516. [PubMed]

6. Joshi HB, Stainthorpe A, MacDonagh RP, Keeley FX, Timoney AG. Indwelling ureteral stents: evaluation of symptoms, quality of life and utility. J Urol. 2003;169 (3): 1065-1069.

7. Dunn MD, Portis AJ, Kahn SA, YAn Y, Sholhav AL, Elbahnasy AM et al. Clinical effectiveness of new stent desingn: randomized single blind comparison of tail and double pig-tails stents. J Endourol. 2000;14(2):195-202.

Correspondencia autor: Dr. A. Vega Vega

Servicio de Urología. Complejo Asistencial de León

Altos de Nava, s/n. 24071 León. Tel.: 987234900

E-mail autor: debgarcia100@hotmail.com

Información articulo: Original - Endourología

Trabajo recibido: octubre 2006

Trabajo aceptado: diciembre 2006 